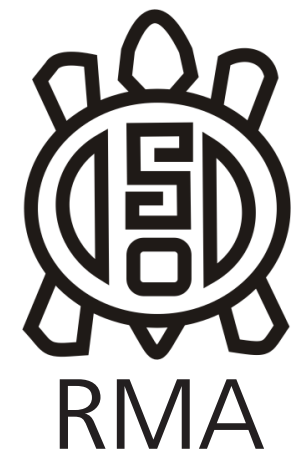

Dossier

\section{Cadena operativa de las puntas bifaciales de Offing 2 - locus 1, estrecho de Magallanes, Patagonia Austral, Chile. Una aproximación tecno-funcional}

\author{
Chaine opératoire of the bifacial points of Offing 2, locus 1, Strait of \\ Magellan, Southern Patagonia, Chile. A techno-functional approach
}

\title{
Resumen
}

Se presentan los resultados del análisis tecnológico y funcional de las grandes puntas lanceoladas fabricadas en los niveles inferiores del sitio de Offing 2, canal Whiteside, Estrecho de Magallanes. Estos artefactos son característicos de las poblaciones marítimas de la región hacia los 4.000 años AP. Los resultados muestran una cadena operativa de fabricación in situ que incluye una formatización por percusión blanda y dura, de una sola vez y en una misma locación, y un acabado por presión, cuyas características (localización, posición, continuidad y cantidad de series) pueden variar. Los resultados funcionales son poco concluyentes, pero son coherentes con una gestión particular de estos artefactos, que involucra conductas de transporte y reavivado. Se propone una hipótesis (a evaluar) de funcionamiento flexible (como arma y cuchillo) asociado a este tipo de gestión.

Palabras clave: Puntas bifaciales; Cadena operativa; Análisis funcional; Sociedades canoeras; Patagonia austral

\begin{abstract}
We present the results of the technological and functional analysis of the large lanceolate points manufactured at the lower levels of the Offing 2 site, Whiteside channel, Strait of Magellan. These artifacts are characteristic of the maritime populations of the region around 4,000 years BP. The results show a reduction sequence which includes shaping by hard hammer and soft hammer percussion, all at once and at the same location, and a final retouch by pressure flaking, the characteristics of which (location, direction, continuity, quantity of removals sharing a same platform) may vary. The functional results are inconclusive, but are consistent with a particular management of these artifacts, involving transport and re-sharpening behaviors. A hypothesis of a flexible functioning (as knives and weapon heads) associated with this type of management is proposed.
\end{abstract}

Keywords: Bifacial points; Chaine opératoire; Use-wear analysis; Canoe societies; Southernmost Patagonia.

Las grandes puntas lanceoladas, denominadas por algunas autoras "tipo Ponsonby", son un artefacto característico de las poblaciones marítimas de Fuego-Patagonia entre los ca. 4.200 y los 3.000 años AP sin calibrar (Legoupil y Pigeot 2009; Morello et al. 2002). Estas puntas se han recuperado en diversos sitios habitacionales costeros en el mar de Otway, el Estrecho de Magallanes y el Canal Beagle; así como de forma aislada en contextos superficiales en localidades de Ultima Esperanza, mar de Otway, isla Dawson y costa noreste de Tierra del Fuego (Legoupil y Pigeot 2009; Morello et al. 2002). Los contextos asociados presentan evidencias de una subsistencia variable, basada en los pinnípedos y en particular los otáridos, aves, peces demersales y guanaco; mostrando además una industria ósea muy elaborada, característica de las poblaciones canoeras, que incluye distintos tipos de cabezales óseos (arpones, azagayas y lanzas multidentadas), cinceles sobre radio de pinnípedo y puntas óseas en hueso de ave (San Román et al., 2016). La aparición de las puntas lanceoladas marcaría una discontinuidad técnica con respecto a las adaptaciones marinas anteriores, ya que a esta innovación se le suman otros elementos, particularmente el cese en la explotación de la obsidiana verde, materia prima lítica dominante en los yacimientos marítimos de la zona del estrecho de Magallanes y los mares interiores entre los 6.500 y 5.200 AP (Morello et al., 2002; Ocampo y Rivas, 2004; San Román et al., 2016).

Estas puntas son artefactos muy estandarizados, de morfología lanceolada y simétrica, delgados y largos, 
de hasta $20 \mathrm{~cm}$ (Legoupil y Pigeot 2009; Morello et al. 2002; Schidlowsky 2001). Se fabrican por formatización ${ }^{1}$ bifacial en una cadena operativa bien diferenciada de la producción de lascas (Pigeot 2003; Schidlowsky 2001). No obstante, se desconoce su funcionamiento y función, información capital para evaluar la razón de sus particularidades morfológicas y eventualmente, las causas de su aparición. Este trabajo pretende realizar aportes a esta problemática, a partir del análisis del material del sitio de Offing 2, locus 1.

\section{El sitio de Offing 2}

Offing 2 es un sitio ubicado en el sureste del islote Offing, una pequeña isla en el Canal Whiteside entre la Isla Dawson y Tierra del Fuego (Figura 1). Este trabajo se enfoca en las ocupaciones de los niveles inferiores, fechados entre $3960 \pm 50$ y $4218 \pm 63$ y correspondientes a un paleosuelo, en el que se recuperaron abundantes artefactos líticos y óseos, principalmente restos de pinnípedos (Legoupil 2011). Los restos recuperados indican que las principales actividades fueron la fabricación de puntas bifaciales y el consumo de pinnípedos, aunque también destacan las actividades de elaboración de industria ósea, en hueso de cetáceo, pinnípedo y ave (Christensen 2012, Legoupil 2012). La industria lítica se compone de 1665 artefactos y está dominada por riolitas y en menor medida, de lutitas disponibles localmente en los depósitos secundarios de las paleoplayas. Estas rocas se utilizaron tanto en operaciones de formatización bifacial, destinadas a la producción de puntas; como en operaciones de desbaste. Estas cadenas operativas por lo general se encuentran diferenciadas (Langlais 2011, 2012).

\section{Metodología}

El análisis tecnológico se realizó separando el material por variedad específica de materia prima, a nivel de las cuales se intentaron remontajes sistemáticamente, pudiendo realizarse 39 remontajes técnicos, con distintos grados de completitud.

Se distinguieron las agrupaciones de materia prima que daban cuenta de actividades de formatización bifacial, entre otras cosas, debido a la presencia de acumulaciones de desechos derivados de esta actividad asociados espacialmente. Estos presentan en general características bastante distintivas que permiten diferenciarlos de las lascas asociadas a operaciones de desbaste (ver Pigeot 2003: 121), incluyendo un perfil curvo, negativos de tendencia centrípeta en el anverso, talones muchas veces preparados por facetado, abrasión y/o regularización del frente de extracción y, en muchos casos, estigmas asociados a la percusión blanda tangencial como probable técnica de aplicación de fuerza (línea posterior del talón

\footnotetext{
${ }^{1}$ En este trabajo utilizamos el término "formatización" como sinónimo del francés "faconnage", una operación de talla lítica en la que la materia se modifica para obtener un solo objeto (Inizan et al. 1995)
}

lisa, labio, bulbo difuso o ausente) ${ }^{2}$. Se registraron distintos atributos morfo-técnicos de las lascas. Las piezas bifaciales fueron además objeto de lecturas diacríticas (Dauvois 1978).

El análisis funcional (ver Huidobro, 2018) se llevó a cabo siguiendo los protocolos clásicos de observación a ojo desnudo, lupa binocular y microscopio metalográfico (Keeley, 1980; Semenov, 1964). Los criterios para la descripción e interpretación de las huellas se basan en el modelo de formación de rastros para rocas heterogéneas, así como en los referentes experimentales publicados para riolitas y cineritas del canal Beagle (Alvarez 2003; Clemente 1997; Clemente et al., 2015; Mansur 1999). Se confeccionó asimismo un referente experimental generalista ${ }^{3}$ en riolita y lutita. En estas materias primas, sobre todo en el caso de la lutita, los micropulidos se desarrollan en forma lenta y poco diagnóstica, por lo que el grado de precisión en la determinación funcional suele ser bajo.

\section{Resultados}

Las actividades de formatización bifacial están representadas en la mayoría de los artefactos líticos del sitio, incluyendo al menos 804 lascas (excluyendo fragmentos y esquirlas) y 20 piezas bifaciales (tablas 1 y 2), cuyo número se reduce a 14 luego de la realización de reparaciones (sensu Ramos y Merenzon 2004). Estas se encuentran en distintos estadios de fabricación (tabla 2) y son muy estandarizadas en cuanto a ancho o espesor, pero no en cuanto a largo, que varía entre 100 y 200 $\mathrm{mm}$. Estas diferencias no están asociadas al estadio de fabricación, ya que son observables tanto a nivel del grupo de preformas, como entre las piezas con acabado total o parcial por presión. En otras palabras, se observan preformas sin acabado de largo menor al de algunas puntas terminadas, con lo cual es posible proponer que esta diferencia métrica es intencional.

Se registró un mínimo de 14 eventos de talla in situ, al interior de los cuales se realizaron varios remontajes físicos. Estos eventos incluyen seis agrupaciones compuestas únicamente por desechos, estando la pieza bifacial (el objetivo de la producción) ausente; ocho agrupaciones de desechos asociados a piezas bifaciales, en distintos estadios de reducción; y cinco puntas bifaciales sin desechos asociados en la misma variedad de roca, pero cercanos espacialmente a concentraciones de lascas de formatización bifacial ${ }^{4}$.

\footnotetext{
${ }^{2}$ Cabe mencionar que no todos los desechos de formatización bifacial presentan estas características, y que muchas veces no fue posible asignar las lascas a una actividad de formatización o desbaste. Por otro lado, la realización de remontajes y de agrupaciones en materias primas específicas también contribuyó a realizar esta clasificación

${ }^{3}$ Es decir, no orientado a la generación de criterios diagnósticos para identificar una actividad específica, sino que incluyendo distintas materias y modos de acción como primera aproximación

4 Las agrupaciones compuestas únicamente por puntas, no se
} 
A.

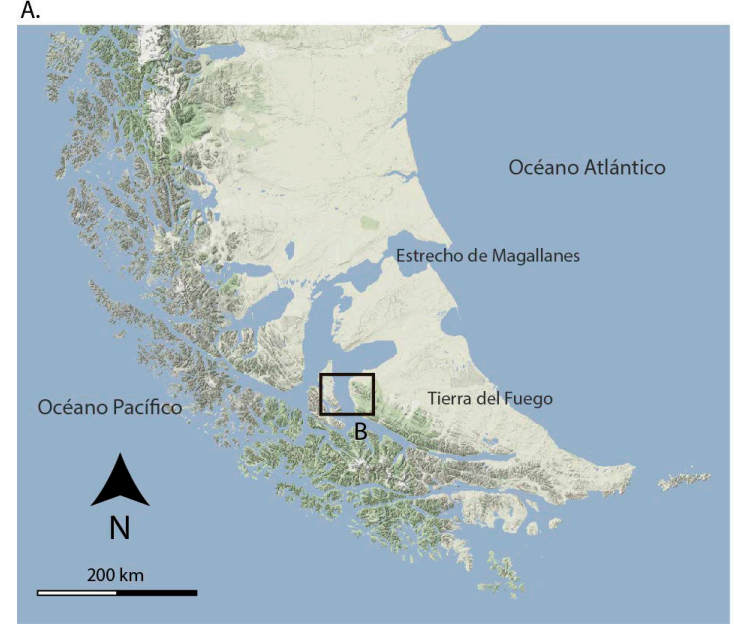

B.

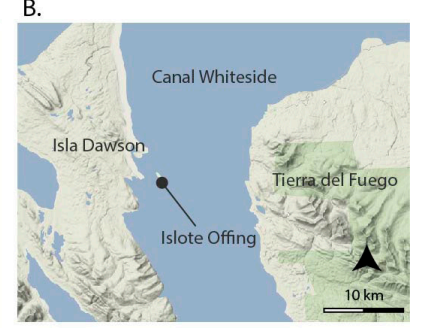

c.

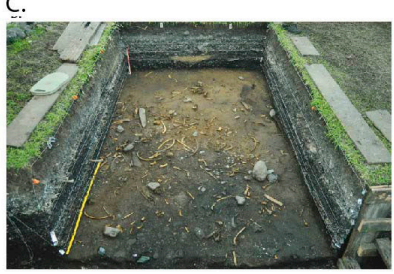

Figura 1: Sitio Offing 2 locus 1. A y B: Ubicación. C: Excavación (foto misión arqueológica francesa).

Figure 1: Offing 2 locus 1 archaeological site. A, B: site location. C: excavation (cl. Mission archéologique française)
Estos artefactos se asocian a dos unidades de actividades, separadas por un sector con menor densidad de material (Figura 2). La distribución de los desechos en estas concentraciones, así como la realización de remontajes, son coherentes con la fabricación de puntas desde un mismo puesto de trabajo hasta terminar la pieza.

Los resultados alcanzados dan cuenta de la representación en el sitio de distintas etapas de la cadena operativa de las grandes puntas lanceoladas. En primer lugar, hay información sobre los soportes utilizados, que serían, al menos en parte, lascas de tamaño grande, probablemente obtenidas fuera del sitio, ya que sus características dimensionales no son coherentes con lo desbastes registrados. Esto está evidenciado en un esbozo inicial, así como en dos preformas en estadios más avanzados de reducción, que presentan porciones de la cara ventral o del talón del soporte. Asimismo, algunas de las lascas de formatización bifacial, muestran, en su cara dorsal, porciones de la cara ventral de la lasca usada como forma base.

Estos soportes, presumiblemente obtenidos fuera del sitio, habrían sido primero preparados por percusión directa dura. Esta fase de fabricación pudo observarse claramente en un remontaje, compuesto de una lasca de gran tamaño de riolita, utilizada como forma base, sobre la que remontan 23 lascas, asociándose además 30 en la misma variedad de materia prima. Sin embargo, este proyecto fue abandonado de forma prematura, debido a la presencia de negativos en bisagra superpuestos que impidieron continuar con la extracción de lascas invasivas. El uso de percusión dura en esta etapa, se encuentra de igual modo atestiguado por la presencia de al menos 29 (tabla 3) lascas que es posible asociar en forma clara a secuencias de formatización bifacial, que presentan atributos diagnósticos de esta técnica (punto de impacto claro, talones espesos, cono, y en algunos casos fisuras asociadas al cono). Ninguna de las preformas ni las lascas

contabilizaron como eventos mínimos de talla, ya que al no tener desechos asociados, no encontramos evidencia de su talla in situ. en esta etapa presenta huellas claras de utilización, con una excepción: una posible preforma inicial, que muestra macro huellas que evocan un uso en percusión, como pieza intermediaria (ver criterios diagnósticos en de La Peña, 2011). Estas huellas corresponden a esquirlamientos bifaciales superpuestos, de morfología trapezoidal y terminaciones abruptas, que forman trituramientos, en uno de los bordes de la pieza, que correspondería a la parte presumiblemente percutida. El borde opuesto presenta esquirlamientos alternantes o parcialmente bifaciales, alineados y superpuestos, semicirculares y trapezoidales con terminaciones abruptas.

La siguiente etapa, una progresión continua de la anterior, es el adelgazamiento y formatización por lascados invasivos obtenidos por percusión blanda, y se encuentra muy bien representada en el sitio. Está evidenciada por numerosas lascas que presentan atributos coherentes con esta técnica (tablas 1 y 3), asociadas espacialmente y que en varios casos remontan entre sí. Tres preformas recuperadas en asociación a estas concentraciones de desechos, parecen haberse fracturado en esta etapa. No presentan ningún indicio de utilización, por el contrario, presentan remanentes de preparación de plataforma por facetado y regularización del frente de extracción, lo que coincide con las características de las lascas. Se encuentran también indicios de aprovechamiento de las lascas obtenidas en esta etapa como soportes de instrumento. Dos lascas tienen retoques marginales de distribución continua parcial (pero no huellas de uso claras) y una, no retocada, presenta huellas de uso en cinemática longitudinal.

La siguiente etapa corresponde al acabado por presión, que permite delinear el borde, dejando una microdenticulación más o menos acentuada. La realización de esta operación in situ está evidenciada por la recuperación de 500 microlascas en una prueba de harneo con agua. Las puntas sin embargo varían en cuanto a la realización de esta operación. Dos piezas muestran un acabado parcial (tabla 2), posiblemente fracturándose en esta etapa por flexión simple. Una de ellas está asociada a desechos 
Tabla 1: Lascas y fragmentos asociados a operaciones de formatización bifacial

Table 1: Bifacial shaping flakes

\begin{tabular}{|c|c|c|c|c|c|}
\hline Categoría & \multicolumn{5}{|c|}{ Materia prima } \\
\hline & Riolita & Lutita & Otras & Indeterminadas & Total \\
\hline Lasca cortical & 8 & & 2 & & 10 \\
\hline Lasca interna & 618 & 79 & 6 & 1 & 704 \\
\hline Esquirla & 545 & & & & 545 \\
\hline Fragmento & 96 & 1 & & & 97 \\
\hline indet & 4 & & & & 4 \\
\hline Total general & 1271 & 80 & 8 & 1 & 1360 \\
\hline
\end{tabular}

Tabla 2: Piezas bifaciales de Offing 2, en distintos estadios de fabricación. Abreviaciones: FPD= Formatización por percusión dura; FPB: Formatización por percusión blanda; TP: Terminación por presión; FRG: fragmento

Table 2: Offing 2 bifaces, showing different production stages. FPD: hard-hammer percussion shaping; FPB: Soft-hammer percussion shaping; TP: pressure retouch; FRG: broken biface

\begin{tabular}{|c|c|c|c|c|c|c|c|}
\hline Identificación & Estado técnico & $\begin{array}{l}\text { Características } \\
\text { técnicas }\end{array}$ & Estado & Largo $(\mathrm{mm})$ & Ancho $(\mathrm{mm})$ & $\begin{array}{c}\text { Espesor } \\
(\mathrm{mm})\end{array}$ & $\begin{array}{c}\text { observacion } \\
\text { es }\end{array}$ \\
\hline D12-450 & Esbozo inicial & FPD & Entera & 114 & 46 & 16 & $\begin{array}{c}\text { Superposici } \\
\text { ón de } \\
\text { negativos } \\
\text { en bisagra }\end{array}$ \\
\hline D13-393 & Esbozo inicial & FPD & Entera & 142 & 54 & 16 & $\begin{array}{c}\text { Superposici } \\
\text { ón de } \\
\text { negativos } \\
\text { en bisagra } \\
\end{array}$ \\
\hline D9-166 & $\begin{array}{l}\text { Esbozo } \\
\text { inicial? }\end{array}$ & FPD & Entera & 147 & 61 & 21 & $\begin{array}{l}\text { Retomada } \\
\text { en uso en } \\
\text { percusión }\end{array}$ \\
\hline $\begin{array}{l}B 7-65+B 7- \\
89+D 7-100\end{array}$ & Preforma & FPB+TP parcial & Reparada & 188 & 38 & 10 & $\begin{array}{c}\text { Retomado } \\
\text { por presión } \\
\text { de uno de } \\
\text { los } \\
\text { fragmentos }\end{array}$ \\
\hline $\begin{array}{c}\text { D12-415+D12- } \\
467+D 13559+ \\
\text { E11598 }\end{array}$ & Preforma & FPB+TP parcial & Reparada & 138 & 35 & 10 & \\
\hline D14-560 & Preforma & FPB & $\begin{array}{c}\text { Frg. } \\
\text { proximal }\end{array}$ & & 47 & 12 & \\
\hline $\mathrm{E} 14-620$ & Preforma & FPB & $\begin{array}{c}\text { Porción } \\
\text { proximal }\end{array}$ & & 30 & 9 & \\
\hline $\begin{array}{c}\mathrm{D} 11417+\mathrm{D} 124 \\
40\end{array}$ & Preforma? & FPB+TP parcial & Reparada & 139 & 30 & 11 & $\begin{array}{c}\text { Retoque por } \\
\text { presión } \\
\text { posterior a } \\
\text { fractura } \\
\text { (impacto?) }\end{array}$ \\
\hline C7-100 & $\begin{array}{c}\text { Punta } \\
\text { terminada }\end{array}$ & $\mathrm{FPB}+\mathrm{TP}$ & Entera & 108 & 28 & 7 & $\begin{array}{c}\text { Esquirlamie } \\
\text { nto distal }\end{array}$ \\
\hline D11-413+414 & $\begin{array}{c}\text { Punta } \\
\text { terminada con } \\
\text { denticulación } \\
\text { acentuada } \\
\end{array}$ & $\mathrm{FPB}+\mathrm{TP}$ & $\begin{array}{c}\text { Porción } \\
\text { distal } \\
\text { reparada }\end{array}$ & & & & $\begin{array}{c}\text { usada en } \\
\text { cinemática } \\
\text { longitudinal }\end{array}$ \\
\hline D13-550 & $\begin{array}{c}\text { Punta } \\
\text { terminada con } \\
\text { denticulación } \\
\text { acentuada }\end{array}$ & & $\begin{array}{l}\text { Porción } \\
\text { proximal }\end{array}$ & & 30 & 8 & $\begin{array}{c}\text { Usada en } \\
\text { cinemática } \\
\text { longitudinal }\end{array}$ \\
\hline D13-540 & $\begin{array}{c}\text { Punta } \\
\text { terminada }\end{array}$ & FPB+TP parcial & $\begin{array}{c}\text { Porción } \\
\text { distal }\end{array}$ & & 26 & 6 & \\
\hline E12-526 & $\begin{array}{c}\text { Punta } \\
\text { terminada con } \\
\text { denticulación } \\
\text { acentuada } \\
\end{array}$ & $\mathrm{FPB}+\mathrm{TP}$ & $\begin{array}{l}\text { Porción } \\
\text { medial }\end{array}$ & & 30 & 9 & $\begin{array}{c}\text { Usada en } \\
\text { cinemática } \\
\text { longitudinal }\end{array}$ \\
\hline B6-1 & $\begin{array}{c}\text { Punta } \\
\text { terminada }\end{array}$ & $\mathrm{FPB}+\mathrm{TP}$ & Entera & 192 & 33 & 9 & \\
\hline
\end{tabular}

Tabla 3: Características técnicas asociadas a lascas de formatización bifacial (se excluyen fragmentos y esquirlas).

Table 3: Bifacial shaping flakes technical attributes (broken flakes and debris are excluded)

\begin{tabular}{|c|c|c|c|c|c|c|}
\hline & \multicolumn{5}{|c|}{ Técnica de aplicación de fuerza (hipótesis) } & \multirow[b]{2}{*}{ Total } \\
\hline $\begin{array}{c}\text { Tipo de } \\
\text { talón }\end{array}$ & $\begin{array}{c}\text { Percusión } \\
\text { dura }\end{array}$ & $\begin{array}{l}\text { Percusión } \\
\text { blanda }\end{array}$ & $\begin{array}{l}\text { Percusión } \\
\text { blanda? }\end{array}$ & $\begin{array}{l}\text { Percusión blanda } \\
\text { orgánica }\end{array}$ & Indet. & \\
\hline cortical & & 2 & & 1 & & 3 \\
\hline facetado & 1 & 48 & & 21 & & 70 \\
\hline filiforme & & 40 & 1 & 3 & & 44 \\
\hline rebajado & & & 1 & 1 & & 2 \\
\hline $\begin{array}{c}\text { Liso } \\
\text { abradido }\end{array}$ & & 250 & & 84 & & 334 \\
\hline liso & 28 & 158 & 5 & 40 & & 228 \\
\hline Indet. & & & & & 54 & 54 \\
\hline Total & 29 & 496 & 8 & 150 & 54 & 736 \\
\hline
\end{tabular}



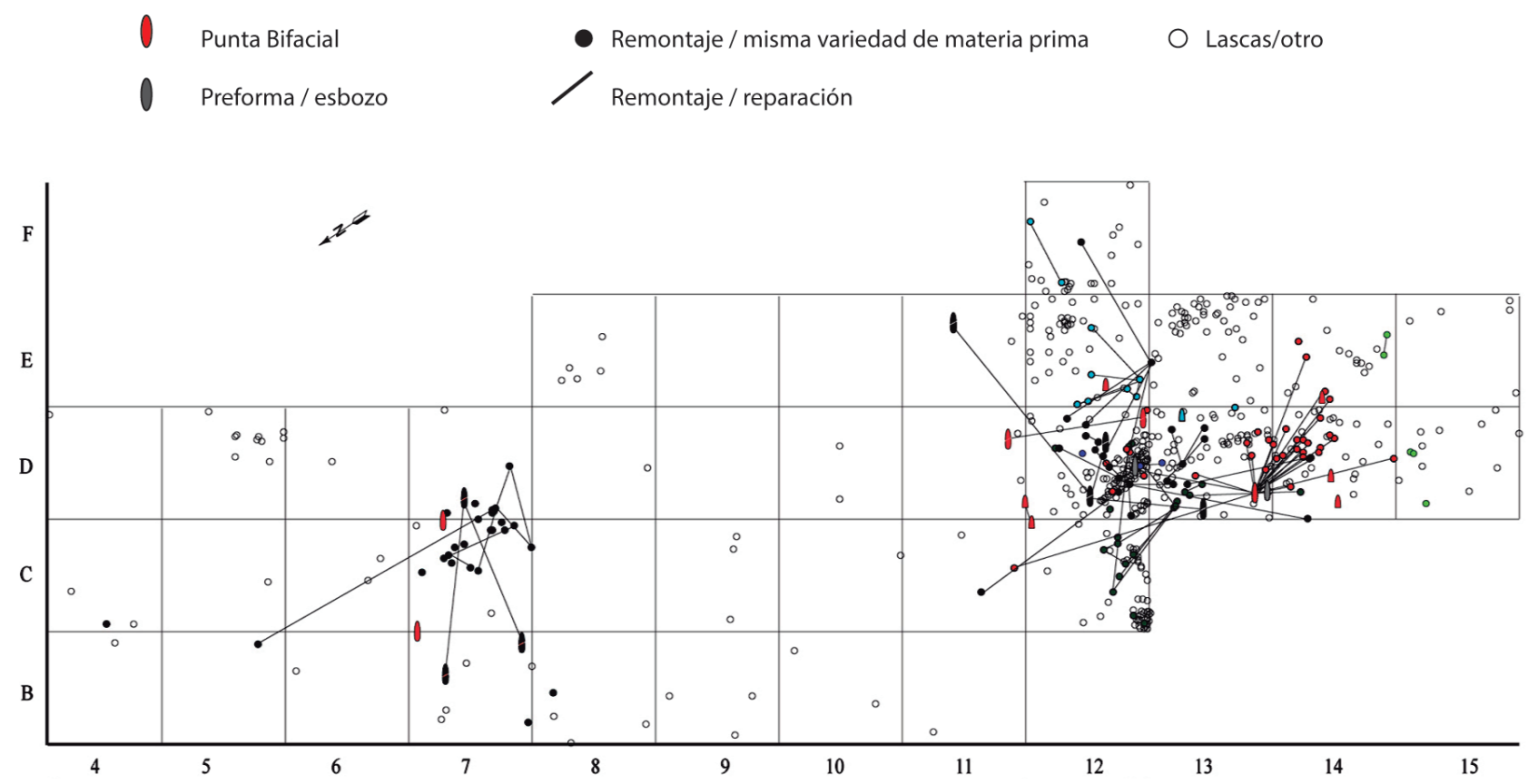

Figura 2: Distribución espacial de los artefactos asociados a formatización bifacial (dibujo M. Langlais)

Figure 2: Spatial distribution of artifacts associated with bifacial shaping (M. Langlais)

en la misma materia prima, y presenta una tentativa de retomado, posiblemente para fabricar una punta de menor tamaño a partir de uno de los fragmentos. No tiene ninguna huella de utilización, y algunas microhuellas técnicas (asociadas a la fabricación) están conservadas.

La segunda es un caso complejo (Figura 3 A). En la parte distal tiene un negativo burilante, de una fractura por flexión simple, sin lengüeta. Luego de esta fractura, la punta fue retomada en uno de sus bordes por presión, lo que parece haber producido la fractura de la pieza y su abandono. Las fracturas burilantes se han asociado experimentalmente al impacto y por lo tanto a una utilización como arma (para una revisión, ver Coppe y Rots 2017); no obstante, el diagnóstico de este tipo de funcionamiento depende de registrar un conjunto de huellas coherentes, macro y microscópicas, así como de identificar asociaciones recurrentes dentro de conjuntos de piezas (Rots, 2016). En este sentido, sobre todo considerando su carácter aislado, este indicio debe tomarse con cautela.
Figura 3: Ejemplos de puntas bifaciales con modificaciones. A. Punta bifacial con fractura distal (negativo burilante y fractura transversal en snap). B. Punta bifacial denticulada con huellas de utilización en cinemática longitudinal.

Figure 3: Modified bifacial points. A. Bifacial point showing distal fractures (burination and snap). B. Denticulated point with use wear showing a longitudinal motion.
A.
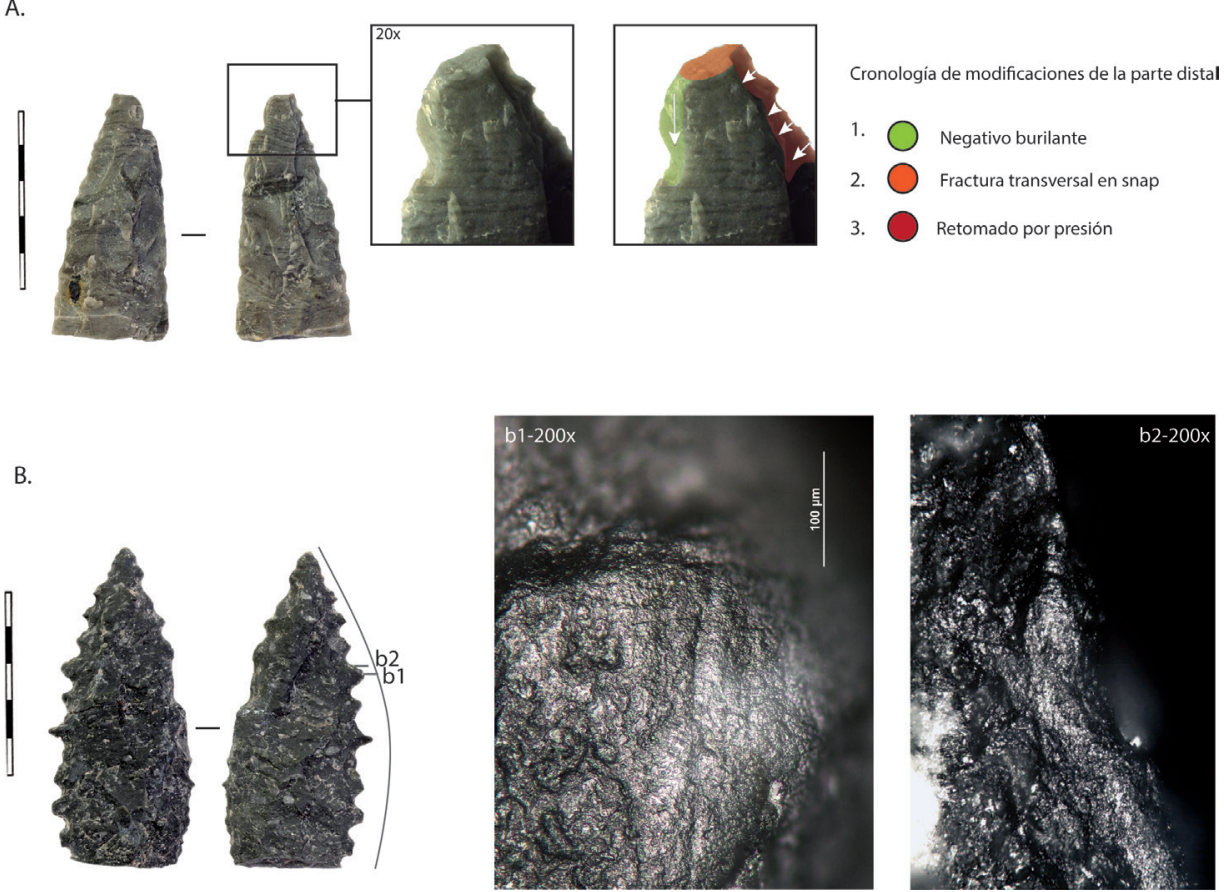

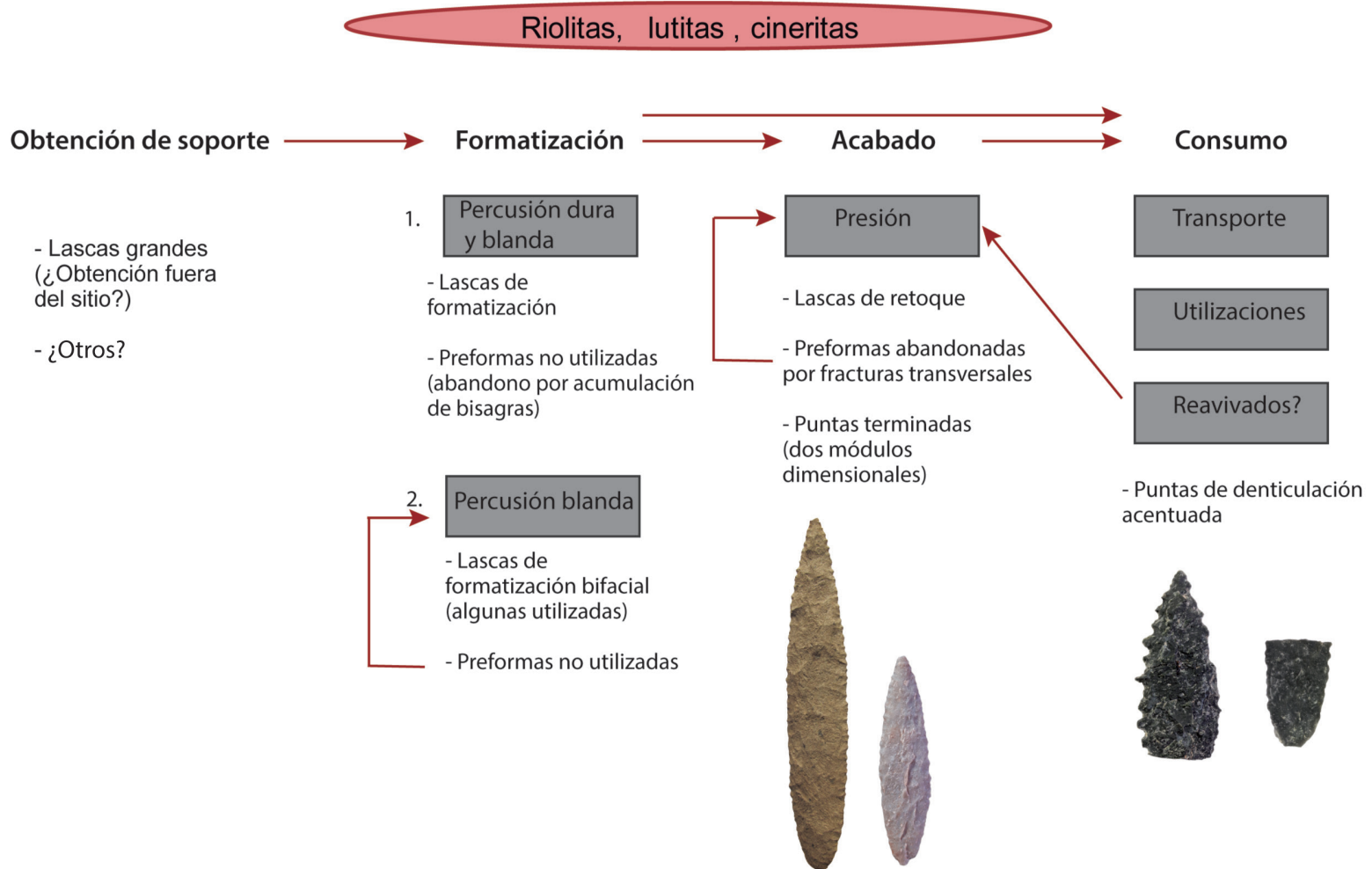

Figura 4: Resumen de las fases de la cadena operativa de fabricación y uso de las punta bifaciales en los niveles inferiores de Offing 2.

Figure 4: Chaine operatoire of the bifacial points represented in the lower levels of Offing 2.

Otras tres piezas presentan un acabado total por presión, bifacial y regular en dos casos y alternante en un tercero; pero sin huellas de utilización claras, además de un ligero esquirlamiento de la punta o fracturas marginales poco diagnósticas. No presentan desechos asociados, salvo un caso, en que se trata únicamente de un microdesecho.

Finalmente, tres puntas se caracterizan por una denticulación acentuada formada por la ejecución de retoques bifaciales utilizando la misma plataforma de presión en ambas caras, lo que a veces se repite en varias series. No obstante, esto no es sistemático ni siquiera a nivel de la misma pieza, ya que también se ejecutan nuevos retoques por presión utilizando la micro-corniza del negativo anterior como plano de presión. Esto mantiene el microdenticulado, pero no lo acentúa. Este grupo de puntas presenta huellas de uso en cinemática longitudinal, en un caso de una materia blanda o media, y no se asocian a agrupaciones de materia prima, aunque sí están descartadas cerca de las acumulaciones de desechos. Las partes salientes están redondeadas y con pequeños esquirlamientos por flexión; pero en general bien conservadas, lo que es un argumento para la dureza relativa, blanda o medianamente blanda, de la materia trabajada. Esto se acompaña de un pulido fluido o granuloso, más desarrollado en los dientes, pero también presente en las concavidades, orientado longitudinalmente (Figura 3 B).

\section{Discusión y conclusiones}

Resumiendo, en Offing 2 se registran distintas fases de la cadena operativa de las grandes puntas bifaciales (Figura 4), incluyendo la reducción inicial de soportes por percusión dura, la formatización por percusión blanda y el acabado por presión. A lo largo de todas estas etapas se produce una gran cantidad de lascas que son solo ocasionalmente recuperadas como soportes de instrumentos, así como preformas descartadas por diferentes accidentes de talla, que no presentan huellas de utilización. La presencia de agrupaciones de lascas de formatización bifacial en la misma materia prima en las que el producto está ausente y lo escaso de los datos funcionales, sugiere que, como es de esperarse ante una hipótesis de uso cinegético, la producción de puntas se realizó para transportarlas fuera del sitio. Hay algunos indicios ambiguos de su utilización como arma y en particular una punta de gran tamaño presenta una fractura compleja, que podría deberse al impacto, posteriormente retomada por presión. Por otro lado, las puntas con denticulación acentuada, utilizadas como "cuchillo", presentan indicios de reactivación y no presentan desechos asociados, lo que sugiere un contexto de uso distinto al de fabricación, y es coherente con una gestión compleja de estos artefactos.

Comparando los pocos datos funcionales existentes para estas puntas (Christensen 2003; Huidobro 2018) es 
posible sugerir un uso polivalente o flexible tanto como arma como cuchillo, lo que es coherente con una de las hipótesis formuladas por Legoupil y Pigeot (2009) en base a las particularidades morfo-métricas de estos artefactos $y$, de forma interesante, con algunos datos etnográficos sobre las armas de cabezal fijo utilizadas por los canoeros (p.e comparar Wallis 1893:171; Weddell, 1827:180-181; King 1839 : 55). Esto debe ser evaluado con un análisis tecno-funcional de la totalidad de las puntas recuperadas (incluyendo las de superficie), y mediante una evaluación experimental contextual, tomando en consideración variables como el sistema de enmangue y modo de propulsión. Asimismo, esto permitiría evaluar la influencia de las particularidades morfológicas de estas puntas en los diferentes tipos de funcionamiento, por ejemplo, los constreñimientos del largo y sobre todo de la relación largo/espesor en un funcionamiento como arma y el eventual rol de la denticulación en un uso como cuchillo/ arma. Al respecto, existen algunos ejemplos etnográficos de las armas denticuladas que señalan un aumento del potencial cortante o para desgarrar, produciendo una herida más intensa (Ellis, 1997). En nuestro caso, el denticulado pudo aumentar la resistencia de los filos y a la vez favorecer su carácter cortante, tal como se ha propuesto en casos arqueológicos similares (Rots et al. 2007). Las materias primas utilizadas en los contextos aquí referidos (riolitas y lutitas) se embotan fácilmente en la utilización, y en particular la riolita es poco adecuada para actividades de corte y descarne. Además, es importante considerar las presas privilegiadas por los canoeros: los mamíferos marinos, que presentan una espesa capa de grasa. Todo esto muestra la importancia de abordar este problema incluyendo evaluaciones experimentales controladas y contextuales, que incluyan estas distintas variables particulares del caso de estudio.

Santiago, 05 de diciembre de 2019

\section{Agradecimientos}

Agradecemos a Dominique Legoupil, Manuel San Román, Ignacio Clemente, Estela Mansur, Flavia Morello y Marianne Christensen. También al equipo de Ethnologie Préhistorique (UMR 7041), por el apoyo económico prestado, y a dos revisores anónimos por sus comentarios y sugerencias.

Estos datos forman parte de un trabajo monográfico en preparación: Langlais, M y Huidobro C en D. Legoupil (dir) Entre Patagonia y Tierra del Fuego: Los nómadas del mar del islote Offing (Isla Dawson, Estrecho de Magallanes) del tercer al primer milenio $A$. C.

\section{Bibliografía}

Alvarez, M. (2003). Organización tecnológica en el canal beagle. El caso de Túnel I (Tierra del Fuego, Argentina). Tesis doctoral, Universidad de Buenos Aires.
Christensen, M. (2003). Analyse fonctionnelle de I'outillage en pierre taillée. En: Les chasseurs-cueilleurs de Ponsonby (Patagonie Australe) et leur environnement du Vleme au Illeme mill. av. J.C (pp. 205-213). Punta Arenas: Magallania, volumen 31

Christensen, M. (2012). Le site d'Offing: I'industrie osseuse, En: D. Legoupil (dir), Le site archéologique d'Offing 2 (locus 1) (détroit de Magellan) étude. Informe Ministerio Francés de Relaciones Exteriores, Ms.

Clemente, I. (1997). Los instrumentos líticos de Tunel VII: una aproximación etnoarqueológica. Barcelona: CSICUAB Treball d'etnoarqueología, 2.

Clemente, I., Lazuén Fernández, T., Astruc, L., \& Rodríguez Rodríguez, A. (2015). Use-wear analysis of nonflint lithic raw materias: The cases of quartz/quartzite and obsidian. En: J. Marreiros, J. Gibaja, y N. Ferreira (Eds.), Use wear and residue analysis in archaeology (pp. 59-81). Manuals in archaeological method, theory and technique.

Ellis, C. (1997). Factors influencing the use of stone projectile tips. An etnographic perspective. En: H. Knecht (Ed.), Projectile technology (pp. 37-74). Nueva York: Springer.

Huidobro, C. (2018). Perspectiva funcional del equipamiento lítico tallado de las sociedades canoeras de magallanes entre los 4.400 - 3.000 años AP. Nuevos resultados a partir del análisis traceológico de Pizzulic 3 y Offing 2 - locus 1 (Componente inferior), Magallania 46(2), 203-230

Keeley, L. (1980). Experimental determination of stone tool uses. London: The University of Chicago Press.

King, F., (1839). Narrative of the surveying voyages of his Majesty's ships Adventure and Beagle between the years 1826 and 1836 describing their examination of the soutjern shores of South America and the Beagle's circumnavigation of the globe (Volumen 1). London: Henry Colbourn, Great Malborough street

Langlais, M. (2011). Les vestiges lithiques-campagne 2011. En: D. Legoupil (dir.) La culture à grandes pointes: Le site archéologique d'Offing 2 (locus 1). Informe Ministerio Francés de Relaciones Exteriores, Ms.

Langlais, M. (2012). La série lithique d'Offing2 (île dawson, détroit de magellan, Patagonie, chili). En: D. Legoupil (dir), Le site archéologique d'Offing 2 (locus 1) (détroit de Magellan) étude. Informe Ministerio Francés de Relaciones Exteriores, Ms.

Legoupil, D. (2003). Cazadores-recolectores de Ponsonby (Patagonia austral) y su paleoambiente desde el VI al III milenio A.C [Número Especial]. Magallania, volumen 31. 
Legoupil, D. (dir) (2011). La culture à grandes pointes: Le site archéologique d'Offing 2 (locus 1). Informe Ministerio Francés de Relaciones Exteriores, Ms.

Legoupil, D. 2012. Les restes alimentaires. Apperçu préliminaire. En: D. Legoupil (dir), Le site archéologique d'Offing 2 (locus 1) (détroit de Magellan) étude. Informe Ministerio Francés de Relaciones Exteriores, Ms.

Legoupil, D. y Pigeot, N. (2009). Les grandes pointes foliacées du type "Ponsonby". Un traceur culturel en Patagonie Australe. Journal de la Société des américanistes, 95(2), 7-32.

Mansur, E. (1999). Análisis funcional de instrumental lítico: problemas de formación y deformación de rastros de uso. En: Actas del XII Congreso Nacional de Arqueología Argentina, La Plata (pp. 355-366).

Morello, F., San Román, M., y Prieto, A. (2002). Puntas de proyectil lanceoladas en Patagonia meridional y Tierra del Fuego. Anales del Instituto de la Patagonia, 30, 155-166.

Ocampo, C y Rivas, P. (2004). Poblamiento temprano de los extremos geográficos de los canales patagónicos: Chiloé e Isla Navarino 1. Chungará 36, 317-331

Pigeot, N. (2003). L'économie de la taille de la pierre. En: D. Legoupil (Ed.), Les chasseurs-cueilleurs de Ponsonby (Patagonie Australe) et leur environnement du Vleme au Illeme mill. av. J.C [Número Especial]. Magallania, volumen 31 (pp. 115-164)

Rots, V., Lentfer, C., Schmid, V. C., Porraz, G., y Conard, N. J. (2017). Pressure flaking to serrate bifacial points for the hunt during the MIS5 at Sibudu Cave ( South Africa ). Plos One, 12(4), 1-61.

San Román, M. (2013). Sitios arqueológicos de la
Isla Englefield, Mar de Otway: Nuevas evidencias de discontinuidad cultural en el proceso de poblamiento maritimo de Patagonia Meridional. En A. F. Zangrando, R. Barberena, A. Gil, G. Neme, M. Giardina, L. Luna, ... A. Tivoli (Eds.), Tendencias teórico-metodológicas y casos de estudio en la arqueología de la Patagonia (pp. 523-534). Mendoza : Sociedad Argentina de Antropología

San Román, M., Reyes, O., Torres, J., y Morello, F. 2016. Archaeology of Maritime Hunter-Gatherers from Southernmost Patagonia, South America : Discussing Timing, Changes and Cultural Traditions During the Holocene. En: H. Bjartmann, H. Mjelva, S. Fretheim, B. Skar, E. Piana y A. Tivoli (Eds), Marine ventures: archaeological perspectives on Human-Sea Relations (pp. 153-170). Sheffield: Equinox Publisher limited

Schidlowsky, V. (2001). Les premiers chasseurs maritimes et les chasseurs terrestres de Patagonie Australe. Comportements techno-économiques et identité culturelle: contributions de la technologie lithique. Oxford: BAR International Series.

Semenov, S. (1964). Prehistoric technology. An experimental study of the oldest tools and artifacts from traces of manufactures and wear. London: Adams \& Dart.

Wallis, S., (1893) Captain Wallis's voyage. In J. Hakesworth, ed. An account of the voyages undertaken by the order of his present majesty for making discoveries in the southern hemisphere and successfully performed by Commodore Byron, Captain Wallis, Captain Carteret and Captain Cook in the Dolphin, the Swallow and the Endeavour pp. 353-649 London: Printed for Strahan \& Cadell

Weddell, J., (1827). Voyage towards the south pole performed in the years 1822-24, London: Longman, Rees, Orme, Brown and Brisbane. 